\title{
Longitudinal Viral Load Monitoring Using Home-Collected Dried Blood Spot Specimens of MSM Living with HIV: Results from a Feasibility Pilot Study
}

\author{
Richard A. Teran ${ }^{1}$ (D) Magdalena E. Sobieszczyk ${ }^{2} \cdot$ Mary Ann Chiasson $^{1,2} \cdot$ Anne-Catrin Uhlemann $^{2,3}$. \\ Jessica Weidler ${ }^{2} \cdot$ Jayesh G. Shah ${ }^{2} \cdot$ Jennifer Y. Chang ${ }^{2} \cdot$ Caitlin Otto $^{4} \cdot$ Sabina Hirshfield ${ }^{5}$
}

Published online: 9 September 2020

๑) Springer Science+Business Media, LLC, part of Springer Nature 2020

\begin{abstract}
Novel viral load monitoring strategies are needed to help individuals maintain an undetectable viral load (UVL). In 2018, U.S. MSM living with HIV with a past detectable VL received a dried blood spot (DBS) kit at baseline and 3-month followup and returned specimens to a research laboratory. Of 56 consenting participants, $91 \%$ returned specimens at baseline and 77\% at 3-month follow-up; 74\% who returned two specimens had UVL at both time points. At-home DBS collection and longitudinal VL monitoring is feasible among U.S. MSM with fluctuating viral load. This complementary approach to clinical care could improve viral suppression maintenance.
\end{abstract}

Keywords HIV $\cdot$ Viral load monitoring $\cdot$ Viral load $\cdot$ Dried blood spots $\cdot$ Feasibility study

\section{RESUMEN}

Se necesitan nuevas estrategias para evaluar los niveles de la carga viral para ayudar a las personas viviendo con VIH a mantener una carga viral indetectable (CVI). En 2018, hombres viviendo con VIH, que reportaron sexo con hombres y una carga viral detectable, recibieron dos paquetes de materiales para colectar muestras de sangre seca después de completar una evaluación basal y después de tres meses de seguimiento. Participantes devolvieron las muestras de sangre seca a un laboratorio. De 56 participantes que dieron su consentimiento a participar en el estudio, el 91\% devolvió una muestra de sangre seca al inicio del estudio y el 77\% a los 3 meses de seguimiento. Además, el 74\% que devolvió dos muestras de sangre seca tenían CVI en ambos puntos de tiempo. La colección de sangre seca en el hogar y el monitoreo longitudinal de la carga viral de VIH es factible entre los hombres viviendo con el VIH y que tienen sexo con hombres. Los resultados de este estudio pueden proporcionar un enfoque complementario a la atención clínica para mejorar la supresión viral del VIH.

Richard A. Teran

richard.teran@columbia.edu

1 Department of Epidemiology, Columbia University Mailman School of Public Health, 722 West 168th Street, 7th Floor, New York, NY 10032, USA

2 Division of Infectious Diseases, Department of Medicine, Columbia University Irving Medical Center, New York, NY, USA

3 Microbiome and Pathogen Genomics Core, Columbia University Irving Medical Center, New York, NY, USA

4 Department of Pathology, SUNY Downstate Health Sciences University, Brooklyn, NY, USA

5 Division of Infectious Diseases, Department of Medicine, SUNY Downstate Health Sciences University, Brooklyn, NY, USA

\section{Introduction}

People living with HIV who are on antiretroviral therapy (ART) and have an undetectable viral load (VL) have no risk of transmitting the virus to HIV-negative partners [1]. Prompt diagnosis of new infections, linkage to care, and ART initiation to achieve and maintain viral suppression is critical to prevent new infections. Novel strategies to monitor VL are needed for people disengaged from HIV care or suboptimally adherent to ART, to understand viral suppression patterns and transmission risk among these populations. eHealth studies with men who have sex with men (MSM) living with HIV report high acceptability of self-collecting biological specimens, including dried blood spots (DBS) $[2,3]$. However, little information exists on longitudinal 
monitoring of VL using self-collected dried blood spots outside of a clinical setting.

In 2016, The Mailed-Spot (M-Spot) study assessed the feasibility of a one-time home self-collection of DBS specimens for laboratory VL quantification [4]. The high uptake and return $(75 \%)$ of a single DBS specimen in the M-Spot study demonstrated the feasibility and acceptability of selfcollection and mailing of specimens to a research laboratory. Among participants who returned a kit with a testable specimen, $53 \%$ had a detectable VL (DVL), and $84 \%$ of DBS specimens with a DVL were from men who self-reported having an undetectable VL (UVL) at their last clinical visit. The current pilot study (the M-Spot 2 study) aimed to determine the feasibility of collecting repeated VL measurements among MSM with a DVL and identify important implementation issues for future studies. We report longitudinal research laboratory-based HIV-1 RNA measurements from MSM living with HIV who self-collected two DBS specimens at home over a 3-month period.

\section{Methods}

The M-Spot 2 study is part of a sequence of studies following participants from the Sex Positive! Study (parent study) and the first M-Spot study [4, 5]. Briefly, eligible participants in the parent study self-reported being biologically male and identified as male or genderqueer; $\geq 18$ years old; White, Black, or Hispanic; able to read and respond in English; a United States (U.S.) resident; living with HIV; virally unsuppressed ( $\geq 200$ copies $/ \mathrm{mL}$ ) in the past year or reported past-month suboptimal ART adherence; and reported condomless anal sex (CAS) with an HIV-discordant or unknown status male partner in the past 6 months.

Men who returned a DBS specimen with a VL above the limit of quantification $(\geq 832$ copies $/ \mathrm{mL}$ ) of the research assay during the first M-Spot study (conducted in 2016) received a recruitment email in 2018 with a link redirecting them to a brief, secure screening survey. Individuals with a history of hemophilia or on anticoagulants were excluded. Consenting participants were mailed a package at baseline and 3-months containing: one HemaSpot ${ }^{\mathrm{TM}}{ }_{-} \mathrm{HF}$ device (DBS kit; Spot On Sciences, Inc., Austin, TX), collection materials, instructions, and a return envelope with postage. At both time points, men also completed a brief web-based survey about their experience using the kit, recent sexual behavior, engagement in HIV care (e.g., VL test results), ART adherence (e.g., suboptimal adherence defined as $<90 \%$ based on an ART adherence scale), and drug use.

For this pilot study, a different research assay was used to test DBS specimens than the research assay used for the first M-Spot study [4]. The purpose of the new research assay was to obtain a lower limit of quantification (500 copies $/ \mathrm{mL}$ vs. 832 copies $/ \mathrm{mL}$ ). A validation process was completed by the research laboratory using 13 unique samples and external controls, which were prepared in triplicate to generate $39 \mathrm{DBS}$, plasma, and whole blood samples to validate elution, RNA extraction, and viral RNA measurements using the new research assay.

Upon receipt of specimens from study participants, deidentified DBS specimens were stored for up to 6 months at $-20^{\circ} \mathrm{C}$ before testing, until completion of the research assay validation process. DBS samples were eluted, and RNA was extracted using an RNeasy kit (Qiagen). TaqMan-based Real-time Quantitative PCR research assay, targeting a conserved consensus region on the long terminal repeat region of HIV-1, was used to quantify VL on a StepOnePlus Real-time PCR System (Applied Biosystems), and samples were run in triplicate. The lower limit of quantification (LLQ) for this research-based assay was estimated at 500 copies $/ \mathrm{mL}$. The research laboratory reported DBS VL results as 'undetectable' if no HIV-1 RNA was detected. A qualitative result of ' $\leq 500$ copies/mL' was reported if HIV-1 RNA was detected below the LLQ. Quantitative VL results were reported by the research laboratory when HIV-1 RNA was detectable above 500 copies $/ \mathrm{mL}$.

The institutional review boards at Public Health Solutions and Columbia University in New York, NY, approved all study-related and laboratory-related procedures, respectively. All participants completed an online informed consent process. After agreeing to participate, participants could select the option (yes/no) to receive their VL results (detectable or undetectable-quantitative results were not shared with participants) over the phone and were asked to provide a phone number. At the end of the study, two phone call attempts (1-2 weeks apart) were made to each participant requesting their DBS specimen results. If participants did not answer either call or return the voicemail message, a text message was sent requesting a callback.

Univariate analyses using frequencies and proportions or medians and interquartile ranges (IQR) were used to describe the distribution of sociodemographic, sexual behavior, engagement in HIV care, ART adherence, and drug use at baseline and 3-month follow-up. We assessed study feasibility by the proportion of participants who successfully collected a testable blood specimen and returned it to the research laboratory at baseline and 3-month follow-up. Participants' characteristic differences stratified by DBS specimen HIV-1 RNA result (detectable vs. undetectable) were assessed at each time point. Chi-square and Fisher's exact tests were used to assess differences in categorical variables, while Wilcoxon Mann Whitney tests assessed differences among continuous variables. Data analyses were performed using SAS version 9.4 (SAS Institute, Cary, NC). 


\section{Results}

From April to May 2018, 78 men who returned a specimen with DVL $\geq 832$ copies/mL during the first M-Spot study were invited to participate in the M-Spot 2 study. Of these, $73 \%$ (56/78) opened the email and clicked on the screener link, provided consent, and enrolled in the study.

Among 56 enrolled participants, 68\% (38/56) were white, $16 \%$ (9/56) were black, and 16\% (9/56) were Hispanic. The median age was 41 years (range: $26-66)$. Over a third $(39 \%$; 22/56) had a college education, $86 \%(48 / 56)$ had private or public health insurance, $39 \%(22 / 56)$ were employed full-time, and $77 \%$ (43/56) reported a yearly income $<\$ 60,000$. HIV care, sexual behavior, and drug use information were collected via an online survey at each time point. A total of 49 and 43 participants completed the survey at baseline and 3-month follow-up, respectively. Most (90\%; 44/49) self-reported being engaged in HIV care, and 67\% (33/49) reported having a $\mathrm{VL}$ test $<6$ months before study enrollment. Among men reporting a recent VL test, 76\% (25/33) self-reported having a UVL ( $\leq 200$ copies/mL). Furthermore, 84\% (41/49) of participants reported current ART use; however, an ART adherence scale indicated that 54\% (22/41) reporting ART use had suboptimal adherence (i.e., $<90 \%$ ), with a median adherence score of $86 \%$ (range: $38-100 \%$ ). Among the 30 men reporting past 30-day CAS with a male partner, 70\% (21/30) reported CAS with an HIV-discordant partner. Drug use history was disclosed by 47 participants, with 40\% (19/47) reporting past 3-month stimulant drug use, and 30\% (14/47) reporting non-stimulant drug use.

Most participants returned a DBS specimen at baseline $(91 \%, 51 / 56)$ and 3-month follow-up (77\%, 43/56). Of the DBS specimens returned at baseline, 43 had UVL, 2 had DVL below the LLQ ( $\leq 500$ copies $/ \mathrm{mL})$, and 6 had quantifiable VL above the research assay's LLQ. Among the 6 baseline specimens with quantifiable VL, the overall median VL was 1475 copies/mL (IQR: 1380-1989 copies $/ \mathrm{mL}$ ). Of note, 3 baseline specimens had DVL $\geq 1500$ copies $/ \mathrm{mL}$ - a threshold described in the literature as the point where HIV transmission risk increases [6]. Of the DBS specimens returned at 3-month follow-up, 36 had UVL, 4 had a DVL below the LLQ, and 3 had quantifiable VL above the LLQ. Among the 3-month follow-up DBS specimens with quantifiable VL, the overall median VL was 1804 copies/mL (IQR: $1525-8257$ copies/mL). Two follow-up specimens had DVL $\geq 1500$ copies $/ \mathrm{mL}$.

Participants also reported the result of their most recent clinical VL test at each time point. At baseline, $63 \%$ (32/51) of participants who returned a DBS specimen reported having a clinical VL test in the past 6 months. Many of these participants $(75 \%, 24 / 32)$ reported having an undetectable VL based on their last clinical VL test; however, one participant had a discordant DVL DBS specimen result. At 3-month follow-up, 49\% (21/43) of participants who returned a DBS specimen reported having a clinical VL test in the 3-month period between returning the baseline DBS specimen to the research laboratory and completing the 3-month DBS follow-up specimen collection. Among this subset, most participants $(71 \%, 15 / 21)$ reported having an UVL based on their last clinical VL test; one participant had a discordant DVL DBS specimen result. Additionally, differences between men returning a specimen with DVL or UVL were assessed at baseline and follow-up. A greater proportion of men with DVL at baseline $\left[60 \%(3 / 5)\right.$ vs. $10 \%(4 / 42), \chi^{2} 8.98$, Fisher exact test $\mathrm{p}=0.019]$ and at 3-month follow-up [57\% (4/7) vs. 3\% $(1 / 35), \chi^{2} 16.39$, Fisher exact test $\left.\mathrm{p}=0.002\right]$ reported not being on ART, compared to men with UVL.

Distinct VL and HIV care visit trajectories were identified among the 51 participants who returned at least one DBS specimen; 63\% (32/51) returned specimens with UVL at both baseline and 3-month follow-up (Fig. 1Group A). Among these men, 26 reported an HIV care visit $\leq 6$ months before enrollment or between enrollment and receipt of baseline study materials, and 18 reported an HIV care visit in the 3-month period between baseline and follow-up.

Among the other specimens received, 5 returned a baseline specimen with UVL and a follow-up specimen with DVL (Fig. 1-Group B). In this group, four reported an HIV care visit $\leq 6$ months before enrollment, and two had an HIV care visit between baseline and follow-up. Four returned a baseline specimen with DVL and a follow-up specimen with UVL (Fig. 1-Group C); of which one reported an HIV care visit before receipt of baseline study materials and two had an HIV care visit between baseline and follow-up. A total of two men returned a baseline and follow-up specimen with DVL (Fig. 1-Group D); neither of them reported HIV care visits before baseline, but both reported an HIV care visit between baseline and follow-up. Additionally, six returned a baseline specimen with UVL, and two men returned a baseline specimen with DVL but were lost to follow-up (Fig. 1-Group E and F).

Among the 51 participants who returned at least one DBS specimen to the research laboratory, $75 \%(38 / 51)$ opted to receive their DBS specimen HIV-1 RNA results over the phone. Over a third of participants $(39 \%, 15 / 38)$ answered their phone or called the research staff after receiving a voicemail or text message notifying them that their results were ready. Most participants $(80 \%, 12 / 15)$ who received their results over the phone returned two DBS specimens with UVL, and 20\% (3/15) returned one specimen with DVL. 


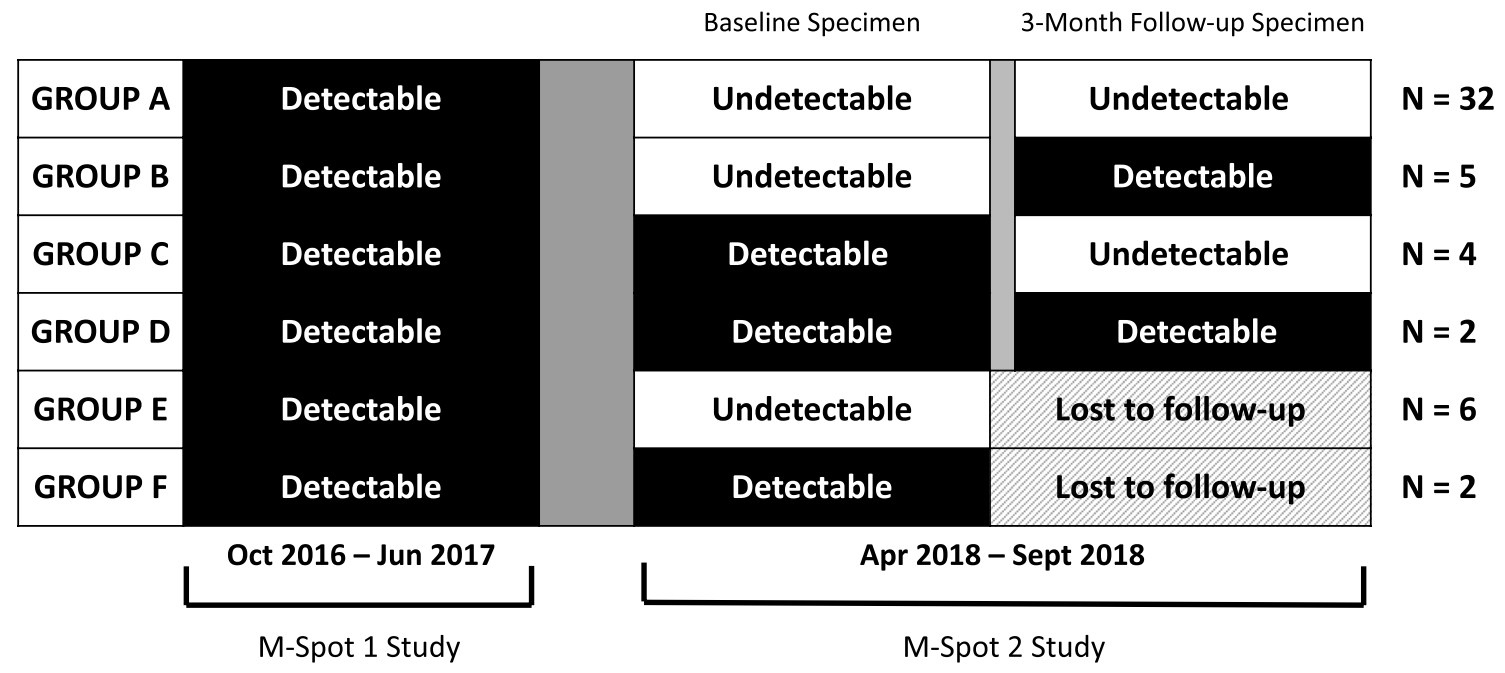

Fig. 1 Dried Blood Spot specimen viral load results among M-Spot 2 participants $(\mathrm{N}=51)$

\section{Discussion}

The M-Spot 2 study assessed the feasibility of collecting multiple DBS specimens for VL quantification in a research setting from U.S. MSM living with HIV who had previously reported suboptimal ART adherence or a DVL, and condomless anal sex with HIV-discordant partners. Feasibility was demonstrated, as $91 \%$ of men provided a testable DBS specimen at baseline, and $77 \%$ provided a testable specimen at 3-month follow-up. Additionally, this pilot study also highlighted critical implementation issues that should be addressed if researchers aim to explore the utility of athome DBS VL monitoring in future studies.

We identified fluctuating VL over 3 months in a subset of participants, indicating non-durable viral suppression. Due to the dynamic nature of HIV care, where individuals can move in and out of viral suppression due to ART nonadherence or disengagement from HIV care, it is important to assess viral suppression over time. The Centers for Disease Control and Prevention currently estimates that $61.5 \%$ of people living with HIV had suppressed VL in 2016 [7]. However, reports indicate that one-third of individuals who achieve viral suppression do not remain suppressed for more than 12 months [8]. A promising finding from our pilot study is that individuals with fluctuating viremia are willing to participate in a research study to measure their VL over time outside of a clinical setting. DBS collection using the methods described above can help researchers monitor VL fluctuations and, if validated in larger studies, provide a complementary monitoring method to standard of care to identify those who struggle to maintain a suppressed VL and may need additional services and support.

A continued area of concern from the first M-Spot study and this pilot study is the number of participants with a discrepant self-reported viral suppression status and DBS specimen result. In the first M-Spot study, the majority of men $(84 \%)$ who returned a DBS specimen with DVL, selfreported an UVL result during their last clinical visit [4]. While the proportion of disagreement was lower in this pilot study, instances of discordant self-reported viral suppression status and DBS specimen laboratory results were seen. This issue has important implications for continued HIV transmission and prevention campaigns such as the Undetectable $=$ Untransmittable movement, which emphasizes that sexual HIV transmission is not possible between HIV-discordant partners when the HIV-positive partner is virally suppressed [1]. To decrease new HIV infections at the population-level, the majority of PLHIV not only need to maintain viral suppression, but their perceived viral suppression status must match their true viral suppression status. Consistent VL monitoring is key to continued prevention efforts and progress towards ending the HIV epidemic. Future studies should continue assessing differences between perceived viral suppression status and DBS specimen VL results. Individuals disengaged in care may be encouraged to return to clinical care if they learn about a detectable VL result from a self-collected DBS specimen.

This pilot study also highlighted several important implementation issues that researchers should consider for future studies, including shipping costs, long-term follow-up, the timing of DBS testing, and sharing results with participants. DBS collection materials and a return envelope were mailed to participants across the U.S. via the U.S. Postal Service for a minimal expense (approximately $\$ 6$ per specimen each way). Future studies incorporating at-home DBS self-collection will need to consider various factors when deciding on a specific shipping protocol, including selecting discrete shipping materials, speed of shipping delivery to and from study participants, 
and management and tracking of return shipping labels. Additionally, while this study demonstrated pilot-feasibility of collecting two DBS specimens over time, future studies should assess if longer-term follow-up and collection of more than two DBS specimens are possible for those suboptimally engaged in HIV care. Furthermore, it is important to note that fiscal issues prevented us from testing DBS specimens as soon as they were received by the research laboratory. Future studies should consider testing specimens in real-time to provide researchers and study participants with VL results within a reasonable window period. Lastly, contacting participants and sharing results presented a unique implementation issue in this pilot study, given that participants were all recruited online, and study procedures were completed at home with minimal interaction with research staff. Although study participants were informed that they would be contacted via a telephone call, most participants did not answer phone calls or return voicemail messages. Future studies should explore alternative ways of contacting study participants, including SMS messages, email, or mobile phone push notifications via a mobile application.

This study had several limitations that should be considered when interpreting our results. First, the study population was recruited from a sample of men who completed a 12-month online intervention and had already provided a testable DBS specimen in a prior study. These participants may have been more willing to enroll in this subsequent pilot study, and different participation rates may be observed in other populations. Second, participants were recruited using email addresses provided in the first iteration of the M-Spot study. Unenrolled participants may have never seen the recruitment solicitation in their inbox or spam folder, which potentially affected enrollment rates. Third, there is concern about the stability of HIV serologic markers in DBS samples, and the correlation of VL results from research-based assays with VL results from plasma samples collected in clinical settings. However, several DBS studies have reported a high correlation between VL results from DBS specimens and those obtained from plasma, and the stability of DBS specimens under different storage conditions for VL quantification and monitoring [9, 10]. Additionally, compared with traditional DBS sampling using Whatman 903 cards, the HemaSpot-HF device used in this pilot study simplifies sample collection and processing as well as improves the stability of specimens [11]. Lastly, the applicability of the research-based extraction and quantification of HIV-1 RNA needs to be further assessed in a larger setting.

\section{Conclusions}

To our knowledge, this is the first study to assess the feasibility of longitudinal VL monitoring via DBS specimens in a research setting, especially among vulnerable populations with suboptimal ART adherence and self-reported HIV transmission risk behavior. New approaches to VL monitoring are needed, and further assessment of these methods in a clinical care setting is warranted. The need for alternative VL monitoring approaches is critical, given the COVID-19 pandemic and reports of shelter-in-place orders disrupting engagement in care [12]. Alternative VL monitoring methods may complement virtual clinical visits and aid clinicians during future public health crises.

There is great potential to use at-home DBS specimen collection in conjunction with HIV clinical care to identify individuals who cannot maintain viral suppression between clinical visits or who disengage in care and are unable to continue on a regular VL monitoring schedule. These individuals need additional services to help them maintain viral suppression and prevent HIV transmission. Future research should assess if individuals with a history of suboptimal ART adherence, or fluctuating VL, may benefit from more frequent VL monitoring between clinical visits via at-home DBS monitoring techniques. Additional VL monitoring may also improve visit attendance rates, engagement in care, and ultimately may improve the number of individuals who achieve and maintain viral suppression.

Acknowledgements This research was supported by a grant from the National Institute of Mental Health (R01-MH100973-03S1) to Sabina Hirshfield, principal investigator, and a grant from the National Institute of Allergy and Infectious Diseases (UM1 AI069470) to Magdalena Sobieszczyk. Richard Teran was supported by a T32 pre-doctoral fellowship in the Global HIV Implementation Science Research Training program sponsored by the Mailman School of Public Health, ICAP at Columbia University, with funding from the National Institute of Allergy and Infectious Diseases (T32 AI114398, PI: Howard). We thank all study participants for their involvement in the study.

Author Contributions Study design (RAT, MES, MAC, and SH), assay validation and specimen testing (MES, A-CU, JW, JGS, and JYC), data analysis (RAT and SH), drafting of article (RAT), critical revision of article (all authors), and final approval of submitted version (all authors).

Funding This research was supported by a grant from the National Institute of Mental Health (R01-MH100973-03S1) to Sabina Hirshfield, principal investigator, and a grant from the National Institute of Allergy and Infectious Diseases (UM1 AI069470) to Magdalena Sobieszczyk.

\section{Compliance with Ethical Standards}

Conflicts of Interest The authors declare that they have no conflict of interest. 


\section{References}

1. Eisinger RW, Dieffenbach CW, Fauci AS. HIV viral load and transmissibility of HIV infection: undetectable equals untransmittable. JAMA. 2019;321(5):451-2.

2. Grov C, Cain D, Whitfield TH, et al. Recruiting a U.S. national sample of HIV-negative gay and bisexual men to complete athome self-administered HIV/STI testing and surveys: challenges and Opportunities. Sex Res Social Policy. 2016;13(1):1-21.

3. Ricca AV, Hall EW, Khosropour CM, et al. Factors associated with returning at-home specimen collection kits for HIV testing among internet-using men who have sex with men. J Int Assoc Provid AIDS Care. 2016;15(6):463-9.

4. Hirshfield S, Teran RA, Downing MJ, et al. Quantification of HIV-1 RNA among men who have sex with men using an at-home self-collected dried blood spot specimen: feasibility study. JMIR Public Health Surveill. 2018;4(4):e10847.

5. Hirshfield S, Downing MJ Jr, Chiasson MA, et al. Evaluation of sex positive! A video ehealth intervention for men living with HIV. AIDS Behav. 2019;23(11):3103-18.

6. Marks G, Gardner LI, Rose CE, et al. Time above 1500 copies: a viral load measure for assessing transmission risk of HIV-positive patients in care. AIDS. 2015;29(8):947-54.

7. Centers for Disease Control and Prevention. Monitoring Selected National HIV Prevention and Care Objectives by Using HIV Surveillance Data United States and 6 Dependent Areas, 2017. 2019. https://www.cdc.gov/hiv/pdf/library/reports/surveillance/ cdc-hiv-surveillance-supplemental-report-vol-24-3.pdf. (Accessed July 28 2019).

8. Marks G, Patel U, Stirratt MJ, et al. Single viral load measurements overestimate stable viral suppression among HIV patients in care: clinical and public health implications. J Acquir Immune Defic Syndr. 2016;73(2):205-12.

9. Garrido C, Zahonero N, Corral A, et al. Correlation between human immunodeficiency virus type 1 (HIV-1) RNA measurements obtained with dried blood spots and those obtained with plasma by use of nuclisens EasyQ HIV-1 and Abbott RealTime HIV load tests. J Clin Microbiol. 2009;47(4):1031-6.

10. Marconi A, Balestrieri M, Comastri G, et al. Evaluation of the Abbott Real-Time HIV-1 quantitative assay with dried blood spot specimens. Clin Microbiol Infect. 2009;15(1):93-7.

11. Manak MM, Hack HR, Shutt AL, et al. Stability of Human Immunodeficiency Virus serological markers in samples collected as HemaSpot and Whatman 903 Dried Blood Spots. J Clin Microbiol. 2018;56(10):e00933-18.

12. Ridgway JP, Schmitt J, Friedman E, et al. HIV care continuum and COVID-19 outcomes among people living with HIV during the COVID-19 pandemic, Chicago, IL. AIDS Behav. 2020;24(10):2770-2.

Publisher's Note Springer Nature remains neutral with regard to jurisdictional claims in published maps and institutional affiliations. 